\title{
Targeting Three-Dimensional Genome Architecture Might Be One of the Mechanisms of Chloroquine's Diverse Therapeutic Actions
}

\author{
Gao-De Li \\ Chinese Acupuncture Clinic, Liverpool, UK \\ Email: gaode_li@yahoo.co.uk
}

How to cite this paper: Li, G.-D. (2020) Targeting Three-Dimensional Genome Architecture Might Be One of the Mechanisms of Chloroquine's Diverse Therapeutic Actions. Open Access Library Journal, 7: e6340. https://doi.org/10.4236/oalib.1106340

Received: April 21, 2020

Accepted: May 12, 2020

Published: May 15, 2020

Copyright $\odot 2020$ by author(s) and Open Access Library Inc.

This work is licensed under the Creative Commons Attribution International License (CC BY 4.0).

http://creativecommons.org/licenses/by/4.0/

\section{(c) (i) Open Access}

\begin{abstract}
Chloroquine (CQ) was initially synthesized as an antimalarial agent, but later on, it also shows immunomodulatory, anticancer, and antiviral effects in clinical practice. Although CQ has been used to treat various conditions for more than half century, the underlying mechanisms of its diverse therapeutic actions remain incomplete. In this paper, we hypothesize that targeting three-dimensional genome architecture might be one of the mechanisms of CQ's diverse therapeutic actions. Based on this hypothesis, new approaches to the treatment and prevention of cancer and coronavirus disease 2019 (COVID-19) are proposed.
\end{abstract}

\section{Subject Areas}

Cell Biology, Molecular Biology, Pharmacology

\section{Keywords}

Chloroquine, Hydroxychloroquine, Anticancer, Antivirus, SARS-CoV-2, Coronavirus Disease 2019 (COVID-19), Immunomodulatory Effects, Three-Dimensional Genome Architecture, Plasmodium falciparum Chloroquine-Resistance Marker Protein (Pfcrmp), Genome Architectural Protein

\section{Introduction}

For many decades of clinical use, chloroquine (CQ) seems to have become a cure-all medicine. It was initially synthesized as an antimalarial agent and had played a crucial role in the battle against malaria until the advent of CQ resistance in malaria parasites [1]. Later on, CQ shows a wide range of therapeutic effects in- 
cluding immunomodulatory, anti-inflammatory, anticancer, and antiviral effects and is still used nowadays with its synthetic analogue, hydroxychloroquine (HCQ), to treat systemic lupus erythematosus, rheumatoid arthritis, cancers, viral infections and other diseases. Currently, a new coronavirus named as SARS-CoV-2 has spread around the world, causing heavy death toll. Since no specific medicine available, CQ and HCQ have been tentatively used to treat patients with coronavirus disease 2019 (COVID-19) that is caused by SARS-CoV-2 [2] [3]. We think that further exploring the mechanisms of CQ's diverse therapeutic actions might contribute to the sensible-use of CQ or HCQ for treating cancer and COVID-19 patients.

CQ has been used for more than half century in clinical practice, but the mechanisms of its diverse therapeutic actions remain incomplete. Currently, all of the explanations for the mechanisms of CQ's diverse therapeutic actions mainly focus on CQ's alkalization of food vacuole (in malaria parasites), lysosome and endosome (in other eukaryotic cells including cancer cells), and CQ's other activities on metabolic pathways seem to be the secondary effects of this alkalization [4] [5] [6] [7]. Obviously, no many researches have been done for investigating CQ's activities within the nucleus of eukaryotic cells. We once proposed that the nucleus may be the key site of CQ's therapeutic action [8]. In this paper, we intend to further explore this key site of CQ's action from a different angle.

In recent decades, more and more researches have demonstrated that threedimensional (3D) genome architecture plays a very important role in regulation of gene expression and cellular functions [9] [10]. A recent study demonstrated that a class of anticancer compounds, curaxins can exert their anticancer activities through altering 3D genome architecture [11]. We postulate in this paper that CQ might also be able to target 3D genome architecture in eukaryotic cells. Detailed description about our thoughts is presented in this paper.

\section{Targeting 3D Genome Architecture Hypothesis for CQ's Diverse Therapeutic Actions}

As a supplement to existing mechanisms for CQ's diverse therapeutic activities, we hypothesize that CQ might exert some of its therapeutic activities through altering 3D genome architecture. There are three pieces of indirect evidence to support this speculation. First, CQ can intercalate into double-stranded DNA [12] [13], which might alter $3 \mathrm{D}$ genome architecture. Second, Pfcrmp is a big nuclear DNA-binding protein $(\mathrm{MW} \approx 430 \mathrm{kDa}$ ) and might be the CQ's target in the nucleus [14] [15]. Based on Uniprot's annotation (Q968Y0_PLAFA), Pfcrmp contains a DNMT1-RFD domain that is a potent histone H3 binding domain [16] and 4 coiled coils which might be involved in gene regulation [17]. Therefore, Pfcrmp might function as a genome architectural protein and CQ might alter 3D genome architecture by binding to Pfcrmp. Third, CQ can induce CAGFs in $P$. falciparum, which belong to extrachromosomal DNA (ecDNA) molecules and might be involved in regulating cellular functions by interacting 
with or joining 3D genome architecture during cell cycle progression [18] [19].

There are two types of 3D genome architectures in eukaryotic cells, i.e., chromosomes and non-chromosome architecture. Chromosomes are not involved in gene regulation because they are the structures that are tightly packaged with chromatin fibres and thus have no space for dynamic change to regulate gene expression. Production of chromosomes during cell cycle progression is quite like production of seeds by a plant. Both chromosomes and seeds are packages containing genetic material for passing on to the next generation. The nonchromosome architecture could be named as chromatin configuration that is jointly constructed by entire chromatin fibres from all chromosomes in a eukaryotic cell [20]. Chromatin configuration is the only dynamic 3D genome architecture that regulates gene regulation during cell cycle progression and is involved in cell differentiation and many pathogeneses including oncogenesis [21] [22]. Thus, targeting the 3D genome architecture by CQ or HCQ mainly refers to targeting the gene-regulation-associated 3D genome architecture or chromatin configuration.

Pfcrmp was discovered in $P$. falciparum, but we believe that Pfcrmp's homologue exists in all eukaryotic cells including human cell, which means that CQ or HCQ can target 3D genome architectures in all eukaryotic cells. After CQ binds to Pfcrmp, or Pfcrmp's homologue, chromatin configuration in cells might be slightly altered, which could cause two consequences. One is that the gene expression pattern will be altered, leading to many genes being either up-regulated or down-regulated, which will affect many metabolic pathways and thus might change cellular functions. CQ's diverse therapeutic activities, such as antiviral, immunomodulatory and anti-inflammatory effects might be linked to this cellular function change, for example, CQ might exert its antiviral effect by reducing susceptibility of target cells to virus. Inside the human body, there are many different cell types that might have different changes in their cellular functions after CQ administration, some of which might benefit the human body, others might not or could even harm the human body. This might be the reason why CQ's activities are so diverse and sometimes unpredictable. The other consequence is that the altered 3D genome architecture will become vulnerable to genotoxic factors including intracellular reactive oxygen species (ROS) and anticancer agents. If the cells with this altered $3 \mathrm{D}$ genome architecture receive second assault by DNA-targeting drugs, they will die easily. This might explain why CQ or HCQ alone is not good enough to treat cancer but is a good adjuvant to anticancer medicine. Due to the same reason, CQ could also be a good adjuvant to immunosuppressant or immunomodulatory agent. The main points about our hypothesis are summarized in Figure 1.

Any factor (chemical, physical and biological) that more or less alters 3D genome architecture or chromatin configuration will have genotoxicity. Since cancer is closely associated with abnormal 3D architecture or chromatin configuration [20] [21], it is obvious that carcinogens cause cancer through altering 


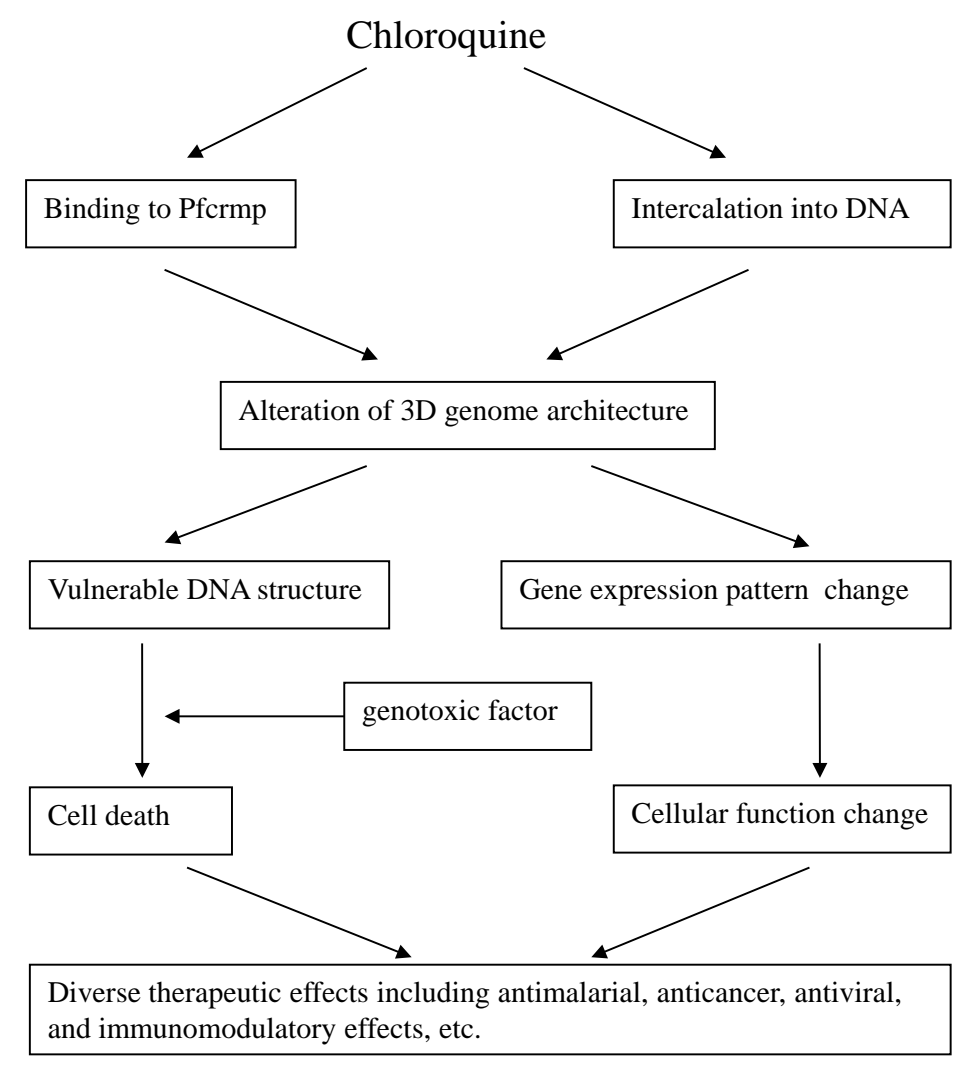

Figure 1. Chloroquine might exert its diverse therapeutic effects by targeting 3D genome architecture in eukaryotic cells.

chromatin configuration. Interestingly, some anticancer drugs that are used to treat cancer can cause cancer [23]. The reason is that anticancer drugs that usually kill both cancer and normal cells can alter chromatin configuration in some survived normal cells and after long-term treatment with the drugs, these cells will eventually become cancer cells. Superficially, genotoxic agents including carcinogens and anticancer drugs are double edged swords, but thinking deeply, genotoxic agent or factor could be a key to eventually solve cancer problem that is tough to cure at present because genotoxic agent is the only agent that could be used to alter chromatin configuration. Hopefully, in future, we can find out a less-toxic genotoxic agent that can alter cancer associated chromatin configuration (CACC) [20], but will not cause normal cells to become cancer cells. In this way, cancer cells might be transformed into normal cells or less harmful cells. Let us conduct an experiment just using imagination, which might support our views described above. First, long-term exposure of normal cells to a low dose of carcinogen $\mathrm{A}$, and the normal cells will eventually become cancer cells. Second, if a high dose of carcinogen A is used to treat these cancer cells, the cancer cells will not be killed because the cancer cells are naturally selected by carcinogen $\mathrm{A}$, which will make the cancer cells become resistant to carcinogen A. Third, if a high dose of carcinogen B that is chemically different from carcinogen A is used to treat these cancer cells, the cancer cells will die because they 
are susceptible to carcinogen B. Last, if a low dose of carcinogen B is used to treat these cancer cells for a quite long time (assuming that this treatment will not cause normal cells to become cancer cells), these cancer cells will become less malignant or become normal cells or be killed by immune system. The reason is that CACC is the worst $3 \mathrm{D}$ genome architecture of cells inside the human body because CACC causes cancer that kills people. Undoubtedly, continuing treatment of cancer cells with different carcinogens will gradually alter CACC and the malignancy of the cancer cells with the altered CACC will be reduced or completely disappears because the malignancy of cancer is determined by CACC and altered CACC is not the original CACC at all. Together, to cure cancer, an inverse process of oncogenesis should be taken, which is a very slow process of altering CACC using a long-term treatment with a low dose of less-toxic genotoxic agent. In this regard, CQ and HCQ could be used for a long time at low dose to treat cancer patients, which might gradually turn cancer cells to normal cells or make cancer cells become less harmful by altering CACC because compared to other genotoxic agents they are less genotoxic and will not cause normal cells to become cancer cells. In future, to prevent cancer, people could be periodically treated with a long course of low dose genotoxic-medicine that will not cause normal cells to become cancer cells but can alter CACC in existing cancer cells. Since most cancers can form and grow undetected for around 10 years, it is better to receive this preventive treatment once every 2-3 years. Hopefully, CQ and HCQ could be used in this way to prevent cancer as well.

Spontaneous remission of cancer (SRC) is a very rare phenomenon, the frequency of which has been estimated to be 1 in 100,000 cancer pateints [24] [25]. The mechanism by which SRC occurs is unknown, but it was suggested that acute infection and fever might be linked to SRC via stimulation of immune system [26]. Based on our hypothesis, SRC might be associated with long-term exposure to very low dose genotoxic factors, of which the cancer patients themselves may be completely unaware. We speculate that fever, viral infection and other factors including pathological and physiological factors might affect dynamic 3D genome architecture in some cells including cancer cells and immune cells inside the patients, which might be used to explain not only the mechanism of SRC but also the reason why CQ's therapeutic activities are different in treatment of different patients with the same condition, for example, currently, whether using CQ or HCQ to treat COVID-19 patients is right or wrong remains arguable [27].

CQ and HCQ might be less genotoxic to most non-dividing cells, but might be more genotoxic to actively dividing cells such as malaria parasites, cancer cells and immune cells etc. Using CQ or HCQ at a low dose for a short period of time might slightly alter chromatin configuration, but the altered chromatin configuration is reversible after withdrawal of the drug. Using CQ or HCQ at a high dose for a long period of time might cause severe genotoxicity and the altered chromatin configuration might become irreversible. CQ resistance in ma- 
laria parasites might be linked to irreversible alteration of chromatin configuration in CQ-resistant malaria parasites, which is caused by long-term treatment with CQ [28]. Compared to other genotoxic drugs, CQ and HCQ are less toxic. However, in order to sensibly use CQ or HCQ for treating various diseases including COVID-19, many factors should be taken into account. Based on our hypothesis, alteration of 3D genome architecture by genotoxic agents including CQ or HCQ could cause both beneficial and detrimental effects to the human body because the response of different cell types inside the human body to the treatment of CQ or HCQ will be different, and more importantly, different pathological and physiological conditions inside the patients also affect the performance of CQ or HCQ. Therefore, to treat COVID-19 patients with CQ or $\mathrm{HCQ}$, assessment of changing patient's conditions and selection of right time to use CQ or HCQ are of great importance.

Based on our hypothesis, a combination therapy using a low-dose CQ or HCQ and a low-dose all-trans retinoic acid (ATRA) could be a safe and effective strategy for treating COVID-19 patients. The purpose of this strategy is to reduce susceptibility of target cells to SARS-CoV-2 and to modulate immune system reaction to SARS-CoV-2 through altering 3D genome architecture in the target cells and immune cells. The reason why ATRA is included in this therapy is based on the assumption that ATRA is directly or indirectly involved in alteration of 3D genome architecture [29] and thus could help to improve the efficacy of CQ or HCQ. In addition, to prevent SARS-CoV-2 infection, administration of these drugs via inhaled route could further reduce drug doses. In conclusion, reducing susceptibility of target cells to virus through altering $3 \mathrm{D}$ genome architecture in the target cells by genotoxic agents is a novel universal-strategy for preventing and treating all viral infections.

\section{Idea for Obtaining Experimental Evidence}

Since chromosome conformation capture technique was invented in 2002 [29] [30], more advanced techniques have been developed to study 3D genome architecture [30] [31]. Therefore, we can use these advanced techniques to study the changes of $3 \mathrm{D}$ genome architecture and gene expression patterns in both CQ treated and untreated cells or malaria parasite ( $P$. falciparum). We can also use CRISPR gene editing technique to modify Pfcrmp or its homologue and then check alterations in both 3D genome architecture and gene expression patterns. Hopefully, in this way, the hypothesis could be tested.

\section{Implications}

If the hypothesis is proven to be true, sensible use of CQ and HCQ in clinical practice will be possible. Besides, further exploration of the underlying mechanism by which CQ alters 3D genome architecture will facilitate developing less-toxic and effective genome-structure-targeting drugs for treating many diseases that have no cure at present. 


\section{Conclusion}

The hypothesis proposed in this paper could be considered as a supplement to existing hypotheses for the mechanisms of CQ's diverse therapeutic actions. There are some pieces of indirect evidence to support this hypothesis, but the direct evidence has to be obtained from experiment. Unfortunately, we don't have any resources to carry out experiment to test this hypothesis, and as such, we just wish that some scientists who are interested in this hypothesis and also have enough resources can test it.

\section{Conflicts of Interest}

The author declares no conflicts of interest regarding the publication of this paper.

\section{References}

[1] Packard, R.M. (2014) The Origins of Antimalarial-Drug Resistance. New England Journal of Medicine, 371, 397-399. https://doi.org/10.1056/NEJMp1403340

[2] Gao, J., Tian, Z. and Yang, X. (2020) Breakthrough: Chloroquine Phosphate Has Shown Apparent Efficacy in Treatment of COVID-19 Associated Pneumonia in Clinical Studies. BioScience Trends, 14, 72-73. https://doi.org/10.5582/bst.2020.01047

[3] Gautret. P., Lagier, J.C., Parola, P., Hoang, V.T., Meddeb, L., Mailhe, M. and Doudier, B., et al. (2020) Hydroxychloroquine and Azithromycin as a Treatment of COVID-19: Results of an Open-Label Non-Randomized Clinical Trial. The International Journal of Antimicrobial Agents, 105949.

https://doi.org/10.1016/j.ijantimicag.2020.105949

[4] Antony, H.A. and Parija, S.C. (2016) Antimalarial Drug Resistance: An Overview. Tropical Parasitology, 6, 30-41. https://doi.org/10.4103/2229-5070.175081

[5] Manic, G., Obrist, F., Kroemer, G., Vitale, I. and Galluzzi, L. (2014) Chloroquine and Hydroxychloroquine for Cancer Therapy. Molecular \& Cellular Oncology, 1, e29911. https://doi.org/10.4161/mco.29911

[6] Plantone, D. and Koudriavtseva, T. (2018) Current and Future Use of Chloroquine and Hydroxychloroquine in Infectious, Immune, Neoplastic, and Neurological Diseases: A Mini-Review. Clinical Drug Investigation, 38, 653-671. https://doi.org/10.1007/s40261-018-0656-y

[7] Duffy. A., Le, J., Sausville, E. and Emadi, A. (2015) Autophagy Modulation: A Target for Cancer Treatment Development. Cancer Chemotherapy and Pharmacology, 75, 439-447. https://doi.org/10.1007/s00280-014-2637-z

[8] Li, G.D. (2006) Nucleus May Be the Key Site of Chloroquine Antimalarial Action and Resistance Development. Medical Hypotheses, 67, 323-326. https://doi.org/10.1016/j.mehy.2006.02.008

[9] Bonev, B. and Cavalli, G. (2016) Organization and Function of the 3D Genome. Nat Rev Genet, 17, 661-678. https://doi.org/10.1038/nrg.2016.112

[10] Li, R., Liu, Y., Hou, Y., Gan, J., Wu, P. and Li, C. (2018) 3D Genome and Its Disorganization in Diseases. Cell Biology Toxicology, 34, 351-365. https://doi.org/10.1007/s10565-018-9430-4

[11] Kantidze, O.L., Luzhin, A.V., Nizovtseva, E.V., Safina, A., Valieva, M.E. and Golov, 
A.K., et al. (2019) The Anti-Cancer Drugs Curaxins Target Spatial Genome Organization. Nature Communications, 10, 1441.

https://doi.org/10.1038/s41467-019-09500-7

[12] O’Brien, R.L., Allison, J.L. and Hahn, F.E. (1966) Evidence for Intercalation of Chloroquine into DNA. Biochimica et Biophysica Acta, 129, 622-624. https://doi.org/10.1016/0005-2787(66)90078-5

[13] Meshnick, S.R. (1990) Chloroquine as Intercalator: A Hypothesis Revived. Parasitology Today, 6, 77-79. https://doi.org/10.1016/0169-4758(90)90215-P

[14] Li, G.D. (2007) Plasmodium falciparum Chloroquine Resistance Marker Protein (Pfcrmp) May Be a Chloroquine Target Protein in Nucleus. Medical Hypotheses, 68, 332-334. https://doi.org/10.1016/j.mehy.2006.07.016

[15] Li, G.D. (2008) Pfcrmp May Play a Key Role in Chloroquine Antimalarial Action and Resistance Development. Medical Hypotheses and Research, 4, 69-73.

[16] Misaki, T., Yamaguchi, L., Sun, J., Orii, M., Nishiyama, A. and Nakanishi, M. (2016) The Replication Foci Targeting Sequence (RFTS) of DNMT1 Functions as a Potent Histone H3 Binding Domain Regulated by Autoinhibition. Biochemical and Biophysical Research Communications, 470, 741-747. https://doi.org/10.1016/j.bbrc.2016.01.029

[17] Matityahu, A. and Onn, I. (2018) A New Twist in the Coil: Functions of the Coiled-Coil Domain of Structural Maintenance of Chromosome (SMC) Proteins. Current Genetics, 64, 109-116. https://doi.org/10.1007/s00294-017-0735-2

[18] Li, G.D. (2016) Certain Amplified Genomic-DNA Fragments (AGFs) May Be Involved in Cell Cycle Progression and Chloroquine Is Found to Induce the Production of Cell-Cycle-Associated AGFs (CAGFs) in Plasmodium falciparum. Open Access Library Journal, 3, e2447. https://doi.org/10.4236/oalib.1102447

[19] Li, G.D. (2017) Cell-Cycle-Associated Amplified Genomic-DNA Fragments (CAGFs) Might Be Involved in Chloroquine Action and Resistance in Plasmodium falciparum. Open Access Library Journal, 4, e3451. https://doi.org/10.4236/oalib.1103451

[20] Li, G.D. (2019) Further Thoughts on Abnormal Chromatin Configuration and Oncogenesis. Open Access Library Journal, 6, e5185.

[21] Corces, M.R. and Corces, V.G. (2016) The Three-Dimensional Cancer Genome. Current Opinion in Genetics \& Development, 36, 1-7. https://doi.org/10.1016/j.gde.2016.01.002

[22] Ibrahim, D.M. and Mundlos, S. (2020) Three-Dimensional Chromatin in Disease: What Holds Us Together and What Drives Us Apart? Current Opinion in Cell Biology, 64, 1-9. https://doi.org/10.1016/j.ceb.2020.01.003

[23] Harris, C.C. (1976) The Carcinogenicity of Anticancer Drugs: A Hazard in Man. Cancer, 37, 1014-1023.

[24] Cole, W.H. (1956) Everson TC. Spontaneous regression of cancer: preliminary report. Annals of Surgery, 144, 366-383. https://doi.org/10.1097/00000658-195609000-00007

[25] Yoon, H.Y., Park, H.S., Cho, M.S., Shim, S.S., Kim, Y. and Lee, J.H. (2019) Spontaneous Remission of Advanced Progressive Poorly Differentiated Non-Small Cell Lung Cancer: A Case Report and Review of Literature. BMC Pulmonary Medicine, 19, 210. https://doi.org/10.1186/s12890-019-0978-4

[26] Jessy, T. (2011) Immunity over Inability: The Spontaneous Regression of Cancer. Journal of natural science, biology, and medicine, 2, 43-49. https://doi.org/10.4103/0976-9668.82318 
[27] Ferner, R.E. and Aronson, J.K. (2020) Chloroquine and Hydroxychloroquine in Covid-19. BMJ, 369, 1432. https://doi.org/10.1136/bmj.m1432

[28] Li, G.D. (2019) Formation of Cell-Type-Associated Chromatin Configurations: A Hypothesis. Open Access Library Journal, 6, e5246. https://doi.org/10.4236/oalib.1105246

[29] Li, Y., He. Y., Liang, Z., Wang. Y., Chen, F. and Djekidel, M.N., et al. (2018) Alterations of Specific Chromatin Conformation Affect ATRA-Induced Leukemia Cell Differentiation. Cell Death \& Disease, 9, 200. https://doi.org/10.1038/s41419-017-0173-6

[30] Dekker, J., Rippe, K., Dekker, M. and Kleckner, N. (2002) Capturing Chromosome Conformation. Science, 295, 1306-1311. https://doi.org/10.1126/science.1067799

[31] Risca, V.I. and Greenleaf, W.J. (2015) Unraveling the 3D Genome: Genomics Tools for Multiscale Exploration. Trends in Genetics, 31, 357-372.

https://doi.org/10.1016/j.tig.2015.03.010 